\title{
Cross-Sectional Surveys as A Tool To Study Epidemiological Factors And Exploring Social Dynamics of Seasonal Influenza In Malta
}

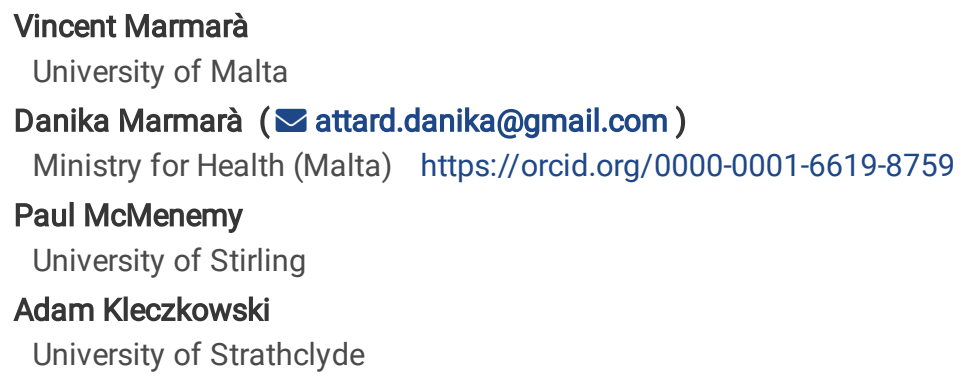

Research article

Keywords: Cross-sectional surveys, under-reporting, seasonal influenza, epidemiology, influenza symptoms, priors

Posted Date: October 16th, 2020

DOI: https://doi.org/10.21203/rs.3.rs-92284/v1

License: (c) (i) This work is licensed under a Creative Commons Attribution 4.0 International License. Read Full License 


\section{Abstract}

Background: Seasonal influenza has major implications for healthcare services as outbreaks often lead to high activity levels in the health system. Mathematical models have extensively been used to predict epidemics of infectious diseases such as seasonal influenza and to assess effectiveness of proposed control strategies. However, the availability of comprehensive and reliable datasets is limited. In this paper we combine a unique epidemiological dataset collected in Malta through GPs with a novel method that uses cross-sectional surveys to study the dynamics of seasonal influenza in Malta in 2014-2016, to include social dynamics and self-perception related to seasonal influenza.

Methods: Two cross-sectional public surveys ( $n=406$ per survey) were performed by phone across the Maltese population in 2014-15 and 2015-16 influenza seasons. Survey results were compared with incidence data (diagnosed seasonal influenza cases) collected by GPs in the same period and with Google Trends data for Malta. The information was collected on whether participants recollected their health status in past months, occurrences of influenza symptoms, hospitalisation rates due to seasonal influenza, whether they sought GP advice, and other medical information.

Results: We demonstrate that cross-sectional surveys are a reliable alternative source of data to medical records. The two surveys gave comparable results, indicating that the level of recollection among the public is high. Based on two seasons of data, the reporting rate in Malta varies between $14 \%$ and $22 \%$. The comparison with Google Trends suggests that the online searches peak at about the same time as the maximum extent of the epidemic, but then the public interest declines and returns to the background level. We also found that the public intensively searched the Internet for influenza-related terms even when the number of cases was low.

Conclusions: Our research shows that a telephone survey is an appropriate way to gain deeper insight into the population self-perception of influenza and its symptoms and to provide another benchmark for medical statistics provided by GPs and Google Trends. The information collected in different ways can be used to improve epidemiological modelling capacity for seasonal influenza and other infectious diseases, thus contributing to public health.

\section{Background}

Seasonal influenza has major implications for healthcare services as outbreaks often lead to high levels of activity in the health system [1]. Such implications continue to emphasize the importance of comprehensive seasonal influenza prediction strategies to health authorities and policy-makers, as well as the importance of pre-planned, accompanying interventions that can be applied across the entire spectrum of healthcare settings. Mathematical models have extensively been used to predict epidemics of infectious diseases such as seasonal influenza and to assess the effectiveness of proposed control strategies [2]. However, for the models to be successfully applied in the public health context, comprehensive and reliable data sets need to be available that accurately describe the incidence. This is not always the case as the reporting rate of such data can be low and they often provide just the number of cases and not the details about the symptoms or patient perception of the severity of the disease [1]. In this paper we introduce a novel method utilising telephone surveys which, to our knowledge, has not yet been used in the context of monitoring infectious disease outbreaks. We then compare the results of the surveys to other sources of epidemiological data for seasonal influenza epidemics in Malta in two seasons: 2014-15 and 2015-16.

There are several definitions of seasonal influenza, but most include the same major symptoms. For example, the UK National Health Service (NHS) states that the symptoms related to seasonal influenza usually develop during the first three days upon becoming infected and include a temperature of high $38{ }^{\circ} \mathrm{C}$ or above, tiredness and weakness, headache, general aches/pains and a dry cough [3]. All of the latter symptoms are similarly defined by the World Health Organization (WHO) [4], but WHO's definition also includes sore throat and rhinorrhea (runny nose). The definition provided by the Centre for Disease Control and Prevention (CDC) is similar to that defined by WHO [5], with the CDC stating that it is common for children to additionally experience vomiting and diarrhoea. The definition used by the NHS has been adopted by the Health Authorities of Malta. It is unlikely that an individual will get infected more than once with the same strain of seasonal influenza during the same season [6], except when an individual does not develop full immunity or when a person is affected by different strains of the seasonal influenza virus [5].

As an important public health problem, seasonal influenza has been addressed with focus on a population's intention to receive future influenza vaccine and associated uptake rates [7-8]; to investigate the knowledge, attitudes and practices of individuals regarding seasonal influenza vaccination [9-12]; to focus on the economic impacts of seasonal influenza, such as the estimation of the direct/indirect costs in relation to outpatient visits and hospitalisation [13]; and on the vaccine and vaccination administration costs [14].

Cross-sectional surveys have performed a principal role in many research fields such as marketing, media and political studies, but their use in epidemiology is still very limited. They have been used to analyse self-perception of the illness $[9,10]$ and attitudes towards influenza vaccination [15]. Surveys relating to influenza can incorporate different methodologies. For example, cross-sectional serological studies are used to explore the response to immunity before and after an influenza outbreak [16], to estimate the proportion of symptomatic infected 
cases [17], and to estimate influenza infection rates [18]. Serological studies are also popularly used in epidemiology to understand various characteristics related to outbreaks as well as the main predictors related to an individual's risks in acquiring the influenza [19]. For example, in a research study by Soh et al. (2012), cross-sectional serological surveys were carried out to estimate the actual infection rates of school-aged children [20]. These surveys have shown that a significant proportion of the population do not visit their General Practitioner (GP) to be examined for their symptoms $[1,21-23]$.

One of the best known recurrent seasonal influenza surveys is the UK flu survey, an online system of monitoring seasonal influenza which forms part of a European project with ten participating countries under the project name InfluenzaNet [24]. Participants are prompted to record and report their symptoms on a weekly basis. Such surveys aim to observe the spread of seasonal influenza through responses over the internet regarding participants' influenza-like illness (ILI) symptoms. Data collected through the UK flu survey have considerable bias towards those individuals that have internet access, with a higher level of education, who are younger in age and who work in an office environment as they have continuous access to the internet. Despite the shortcomings, UK flu survey data are currently used by Public Health England to monitor flu trends in the UK [24]. Since the data are available online, several researchers make use of such information, for example, by Camacho et al. [25] to analyse the duration of cases of ILI and acute respiratory infections (ARI) with their research findings analysed against several demographics. Others used the UK flu survey data to measure ILI and its related risk factors, suggesting that vaccination is linked to the reduced risk of becoming ill with ILI [26].

Online survey data were also used to analyse the incidence rates of seasonal influenza for different locations within the country of Portugal [27]. Similarly, in France [28], researchers analysed real-time data to study the spread of the seasonal influenza disease. In Spain, other researchers made use of their data to compare the incidence rates of countries that are participating in this project [29]. In addition, these Spanish data are being used to understand the mechanisms of the spread of seasonal influenza.

Tan et al. (2013) found that surveys provided useful information about key epidemiological parameters in relation to seasonal influenza [17]. Surveys have also been used to obtain improved and more informative prior distributions [30]. Such prior information can help mathematical models to obtain better forecasts when predicting influenza outbreaks. However, limited research exists about the application of nationwide cross-sectional survey data to improve the understanding of the prior distributions of seasonal influenza outbreaks. Telephone surveys (as used in this study) can offer a good solution to fill missing gaps about knowledge related to the seasonal influenza cases [31].

One of the key limitations for the use of models to describe and predict outbreaks of infectious diseases like seasonal influenza is a gap between the number of actual cases and what can be and is reported [1]. The under-reporting rates - as estimated by serological studies can be very severe, with only a small proportion of actual cases reported to the authorities. In this study we use telephone surveys to gain additional information about seasonal influenza outbreaks in Malta during the 2014/15 and 2015/16 seasonal influenza epidemics. This information is then compared to the doctor reports as well as Google Trends data.

\section{Methods}

Malta is a densely populated country with around 1,311 inhabitants per square kilometre, resulting in a total population of 425,384 [32]. With regards to climate, Malta generally experiences a high humidity level with low variance, except the July-August period characterised by low humidity and temperatures exceeding a daily average of 30 degrees Celsius.

\section{Cross-sectional surveys}

Two cross-sectional surveys were carried out during two different years. The first survey (Survey 1) was carried out between week 35 (end of August 2015) and week 37 (September 2015), and its primary aim was to explore the under-reporting rate of seasonal influenza as compared with the data collected by the GPs during the 2014/2015 seasonal influenza. The questionnaire consisted of 32 questions related to whether participants had experienced seasonal influenza, whether they had any particular symptoms, and included queries on socio-demographic factors. Furthermore, respondents were given a list of symptoms to evaluate whether they had experienced any of these symptoms during the past year. The full questionnaire is available upon request from the corresponding author.

This study was preceded by a pilot survey with a small random sample of 20 individuals to ensure that all questions were clear and comprehensible, as well as to ascertain the practicalities of conducting the telephone survey. The initial results through the pilot study showed that the tool was coherent and could be conducted via telephone so no changes were required. The respondents from this pilot study were not included in the larger study.

A second survey (Survey 2), using the same questionnaire, was carried out during the final stages of the 2015/2016 seasonal influenza. Thus, the second survey was carried out earlier in the influenza season then the first survey, when it might be easier for respondents to remember 
their ILI symptoms. The different timings allow us to analyse the reliability and consistency of surveys, and the implications of the timing of the survey for the results.

The interviews were conducted in Maltese but if participants preferred to answer in English, this option was offered at the start of the survey. Each survey comprised a sample of 406 Maltese individuals from the then eligible population of 349,724 individuals [32]. These were drawn independently for each survey. The eligibility criteria to participate in the study were people residing in Malta, aged 18 years and older. The study was carried out using a $95 \%$ confidence level with a maximum margin of error $+/-4.86 \%$. Telephone numbers were generated at random, through a random number generator (Microsoft Excel). In order to ensure representativeness of the population, both samples were stratified based on the demographics, gender, district and age.

\section{General Practitioners' (GPs) data}

The survey results were compared to the data for GP consultations regarding ILI, which were collected by the Maltese Health Authorities, led by the Malta Health Promotion Department (MHPD) for the years 2014/15 and 2015/16. The average number of GPs reporting the weekly ILI cases for both influenza seasons was 5.7 GPs. For the 2014/2015 influenza season, data are available between week 40 and week 21 , while for the subsequent season between week 41 and week 20.

A number of patients were examined for ILI; these were either diagnosed positive or negative. Those who were diagnosed positive (i.e. acute illness including a number of symptoms with onset during the last 7 days, with measured temperatures of $>38^{\circ} \mathrm{C}$, as defined earlier) by the GPs are represented through all the diagnosed datasets. The number of ILI diagnosed individuals was estimated to be 31,514 during the $2014 / 2015$ season, and 29,090 during the 2015/2016 season. This was calculated by scalling up to the whole population of Malta.

\section{Google Trends}

The results from the survey and from the GP reports were compared with another proxy, Google Trends data. This provided time series data for any keyword searches using the Google search engine. Google Trends provides data that represent the search interest relative to the highest point on the chart for the given region and time. A value of 100 is the peak popularity for the term. A value of 50 means that the term is half as popular. Likewise, a score of 0 means the term was less than $1 \%$ as popular as the peak". Google Trends was used to obtain time series data (September 14 - August 15 and September 15 - August 16) for the key words 'Influenza', 'Flu' and 'Cold' in both Maltese and English language for searches that originated in Malta. However, it does not give the actual number of times that the search was carried out, and so estimates in relation to the severity of influenza are missing. For both seasons (2014/2015 and 2015/2016), January emerged as the highest month for searches related to influenza.

\section{Ethical Considerations}

Ethics approval was obtained from the Psychology Ethics Committee at University of Stirling (28th August 2015). Following an explanation of the main purpose of this research to the participants, individuals were invited to participate in the study through a telephone survey. Participants were given the option to opt out from this research study at any time during the 5-minute telephone survey. Furthermore, respondents were also assured that all the collected information would be processed anonymously and confidentially. Once respondents agreed to participate in survey, none opted out during the data collection.

\section{Results}

\section{Population profile}

The participant details for the two surveys are given in Table 1. Both samples have very similar sample characteristics, and any differences in the sample characteristics between both surveys are within the margin of error of $\pm 4.86 \%$. The demographic variables 'Gender' and 'Age' are comparable with official Maltese statistics at the time when the surveys were carried out. 
Table 1

- Basic sample demographics for Surveys 1 and 2.

\begin{tabular}{|c|c|c|}
\hline & Survey $1(n=406)$ & Survey $2(n=406)$ \\
\hline & \multicolumn{2}{|l|}{ Gender } \\
\hline Female & $51.0 \%$ & $52.2 \%$ \\
\hline \multirow[t]{2}{*}{ Male } & $49.0 \%$ & $47.8 \%$ \\
\hline & \multicolumn{2}{|l|}{ Current Status } \\
\hline Employees & $46.5 \%$ & $45.1 \%$ \\
\hline Pensioners & $21.8 \%$ & $22.8 \%$ \\
\hline Housewives/husbands & $21.5 \%$ & $22.5 \%$ \\
\hline Students & $7.5 \%$ & $8.1 \%$ \\
\hline \multirow[t]{2}{*}{ Unemployed } & $2.7 \%$ & $1.5 \%$ \\
\hline & \multicolumn{2}{|l|}{ Level of Education } \\
\hline Primary & $18.7 \%$ & $19.5 \%$ \\
\hline Secondary & $54.6 \%$ & $52.3 \%$ \\
\hline Diploma & $13.5 \%$ & $14.9 \%$ \\
\hline \multirow[t]{2}{*}{ Tertiary } & $13.2 \%$ & $13.3 \%$ \\
\hline & \multicolumn{2}{|l|}{ Age } \\
\hline $18-25$ & $13.1 \%$ & $12.1 \%$ \\
\hline $26-35$ & $14.3 \%$ & $15.8 \%$ \\
\hline $36-45$ & $15.5 \%$ & $13.6 \%$ \\
\hline $46-55$ & $17.5 \%$ & $15.5 \%$ \\
\hline $46-65$ & $22.4 \%$ & $20.2 \%$ \\
\hline \multirow[t]{3}{*}{$66+$} & $17.2 \%$ & $22.9 \%$ \\
\hline & \multicolumn{2}{|c|}{ Average number of individuals per household } \\
\hline & 2.9 & 2.8 \\
\hline
\end{tabular}

\section{Participants' general medical information}

We found that on average, the participants reported visiting their GP 2.7 times in one year (for both surveys), with the majority visiting their GP twice a year (26.4\%), followed by once a year (18.5\%) and three times a year (16.3\%), with $38.8 \%$ visiting more than three times a year. These visits were related to all health problems, not necessarily seasonal influenza. We also found that $41.2 \%$ of the participants took regular medication due to medical conditions such as asthma, diabetes or heart disorders. In the older age group (66+, 89.9\%), the proportion taking regular medication was significantly higher when compared to the younger generation $\left(\chi^{2}(5)=121.11, p\right.$-value $\left.<0.01\right)$. For those between the age of 18 and 25 years, $17.0 \%$ reported taking regular medication; for those between 26 and 35 years, $8.6 \%$ took regular medication; for those between 36 and 45 years, 22.2\% took regular medication, and for those between 46 and 55, 39.4\% took regular medication. Furthermore, results exceed the 50\% threshold for the age group 56-65 (53.8\%). These results are identical between both surveys (Appendix 1 ).

\section{The seasonal influenza vaccine}

The Maltese Government offers the seasonal influenza vaccine free of charge to some groups of individuals (healthcare professionals, young children, elderly people, chronic disease patients and other employees). Everyone else needs to consult their GP to receive their seasonal influenza vaccination at a cost. According to Survey 1 results, $43 \%$ reported that they had received the flu vaccine, while $55.3 \%$ had not taken the vaccine, and $1.7 \%$ did not remember. Of those who received the vaccine, the $66+$ age group (73.9\%) was the only age group that exceeded $50 \%$ uptake. We found that there is a significant association between the different age groups when compared with the vaccine uptake $\left(\chi^{2}\right.$ $(10)=49.86$, p-value $<0.01)$. This result is due to the Maltese Government's inclusion criteria for the free vaccine. Furthermore, the latter result is similar to England's seasonal influenza vaccine uptake rate for those aged 66+ [156]. Those between 18 and 25 years of age are the least 
likely age group to take the seasonal influenza vaccine (22.6\%), while those between 26 and 65 years the uptake rate varied between $36 \%$ and $46 \%$. These results are similar in both surveys.

The individuals who did not take the seasonal influenza vaccine responded as: 'not interested' (41.1\% of individuals), followed by those who were afraid (24.1\%), and 10.7\% who said that they 'feel sick after taking the vaccine'. Similar reasons were provided for Survey 2.

\section{Influenza-Like IIIness (ILI)}

In this part of the study, respondents were asked whether they had experienced symptoms from a list of ILI symptoms such as fever, cough, sore throat, headaches and others symptoms. Symptoms were individually stated to respondents, allowing the respondents to select each applicable symptom. Respondents were asked to reply to this question retrospectively for the period August $2014-$ July 2015 in Survey 1 (carried out in August 2015) and August 2015 - March 2016 in Survey 2 (carried out in April 2016)

The most common symptoms amongst the participants were 'runny or blocked nose', followed by headache, whilst the least common symptoms were vomiting and chest pain (Table 2). According to Survey 1, 15.4\% of the Maltese population did not suffer from any of the above symptoms during the indicated period (20.0\% - Survey 2). However, this does not necessarily mean that the remaining $84.6 \%$ ( $80.0 \%$ Survey 2) definitely suffered from seasonal influenza. For Survey 2, a higher number of individuals did not experience any of the above symptoms. However, the second survey was based on a shorter time period (since survey 2 was carried out in April, while survey 1 in August).

Table 2

-Individual results for 16 different symptoms.

\begin{tabular}{|lll|}
\hline & Survey $1(\mathbf{n}=\mathbf{4 0 6})$ & Survey $\mathbf{2}(\mathbf{n}=\mathbf{4 0 6})$ \\
\hline & Symptoms & \\
\hline Runny or blocked nose & $61.6 \%$ & $58.4 \%$ \\
\hline Headache & $60.6 \%$ & $55.9 \%$ \\
\hline Sore throat & $54.4 \%$ & $50.5 \%$ \\
\hline Cough & $50.0 \%$ & $48.8 \%$ \\
\hline Sneezing & $45.8 \%$ & $54.7 \%$ \\
\hline Feeling tired or exhausted & $41.1 \%$ & $30.8 \%$ \\
\hline Muscle/joint pain & $34.7 \%$ & $34.5 \%$ \\
\hline Fever & $28.6 \%$ & $25.4 \%$ \\
\hline Loss of appetite & $22.4 \%$ & $11.6 \%$ \\
\hline Watery eyes & $21.2 \%$ & $26.1 \%$ \\
\hline Diarrhoea & $18.0 \%$ & $16.0 \%$ \\
\hline Shortness of breath & $16.7 \%$ & $15.5 \%$ \\
\hline Stomach ache & $15.0 \%$ & $12.1 \%$ \\
\hline Nausea & $13.3 \%$ & $9.1 \%$ \\
\hline Chest pain & $11.6 \%$ & $12.1 \%$ \\
\hline Vomiting & $6.9 \%$ & \\
\hline
\end{tabular}

The above results are sorted in descending order to elicit the most common symptoms amongst the participants from both surveys. Respondents were asked to reply for each symptom.

As reported in Survey 1, the most common month for the above symptoms was January 2015, followed by February 2015 and March 2015. The least common months were August 2014, September 2014 and July 2015 (Table 3). The second survey was carried out earlier (April) when compared to Survey 1 (August), hence respondents for Survey 2 were not able to report their status in the period from April to July. However, in Survey 2, January to March were still the most popular months with the above symptoms. Further details are given in the discussion section, where the above data are compared with the ILI diagnosed cases as reported by the GPs for the 2014-2015 season. 
Table 3

- Months that participants indicated as having any of the symptoms, listed in the questionnaire.

\begin{tabular}{|lll|}
\hline & Survey $\mathbf{1}(\mathbf{n}=\mathbf{4 0 6})$ & Survey $\mathbf{2}(\mathbf{n}=\mathbf{4 0 6})$ \\
\hline August & $0.3 \%$ & $0.0 \%$ \\
\hline September & $0.3 \%$ & $2.3 \%$ \\
\hline October & $5.8 \%$ & $2.1 \%$ \\
\hline November & $6.3 \%$ & $7.3 \%$ \\
\hline December & $10.7 \%$ & $14.6 \%$ \\
\hline January & $18.8 \%$ & $26.2 \%$ \\
\hline February & $15.9 \%$ & $29.5 \%$ \\
\hline March & $14.5 \%$ & $18.0 \%$ \\
\hline April & $10.7 \%$ & $n / a$ \\
\hline May & $7.3 \%$ & $n / a$ \\
\hline June & $6.6 \%$ & $n / a$ \\
\hline July & $2.8 \%$ & $n / a$ \\
\hline Total & $100.0 \%$ & $100.0 \%$ \\
\hline
\end{tabular}

Please note respondents were able to indicate more than one month. The second survey was carried out earlier (April) when compared to Survey 1 (August), hence respondents for Survey 2 were not able to mention from April to July months.

On average, the respondents reported that their symptoms persisted for 9.4 days in Survey 1 and 5.9 days in Survey 2 . The difference between the average number of days for Survey 1 and Survey 2 shows that different seasons might have different characteristics related to seasonal influenza or its symptoms, or reflect different timing of surveys in relation to outbreaks. For people with the symptoms, listed in Table $3,56.5 \%$ (Survey 1) and $55.6 \%$ (Survey 2) claimed that they were restricted to stay at home to recover from their ILI symptoms.

\section{Seasonal influenza}

The previous section analysed symptoms related to the seasonal influenza without mentioning the term 'seasonal influenza' to respondents. In this section, we carry out analysis on responses to questions directly related to the term 'seasonal influenza'. The respondents were asked whether they had seasonal influenza during the past year, without revealing the standard definition. Hence, results here are based either on their own understanding of the symptoms of seasonal influenza, and/or on their doctor's advice. Results from Survey 1 show that $29.8 \%$ of the individuals stated that they had seasonal influenza in the period while for Survey 2, 37.2\% reported having seasonal influenza. Furthermore, in Survey $167.0 \%$ of respondents claimed that they did not acquire seasonal influenza (62.8\% - Survey 2$)$ and $3.2 \%$ were unsure (0\% - Survey 2$)$. The most common month for the seasonal influenza according to the respondents in both surveys was January 2015 , followed by February 2015 and December 2014 (Table 4). 
Table 4

- Months in which participants indicated having the seasonal influenza.

\begin{tabular}{|lll|}
\hline & Survey $\mathbf{1}(\mathbf{n = 4 0 6})$ & Survey $\mathbf{2}(\mathbf{n}=\mathbf{4 0 6})$ \\
\hline August & $0.0 \%$ & $0.0 \%$ \\
\hline September & $0.0 \%$ & $0.6 \%$ \\
\hline October & $8.2 \%$ & $0.6 \%$ \\
\hline November & $6.0 \%$ & $7.5 \%$ \\
\hline December & $16.4 \%$ & $17.3 \%$ \\
\hline January & $28.4 \%$ & $34.7 \%$ \\
\hline February & $23.0 \%$ & $30.6 \%$ \\
\hline March & $14.2 \%$ & $8.7 \%$ \\
\hline April & $2.2 \%$ & $n / a$ \\
\hline May & $1.1 \%$ & $n / a$ \\
\hline June & $0.5 \%$ & $n / a$ \\
\hline July & $0.0 \%$ & $n / a$ \\
\hline Total & $100.0 \%$ & $100.0 \%$ \\
\hline
\end{tabular}

Please not respondents were able to indicate more than one month. The second survey was carried out earlier (April) when compared to survey 1 (August), hence respondents for survey 2 were not able to mention from April to July months.

As for symptoms, respondents were allowed to report more than one month for having the seasonal influenza. Those who claimed they had seasonal influenza also claimed to have contracted seasonal influenza an average of 1.50 (Survey 1) and 1.28 (Survey 2) times during the year. There can be several reasons for this effect. Some people with a lower immune system might suffer from seasonal influenza more than once [5] or might suffer from influenza A (which is the common strain of seasonal influenza) and influenza B [33]. However, most people reporting seasonal influenza more than once in a year would have misinterpreted their ILI symptoms as another case of seasonal influenza.

We also asked people for the duration of their seasonal influenza. The respondents claimed that on average the duration of their seasonal influenza was 9.9 days (Survey 1 ) and 9.5 days (Survey 2). This is similar to generally accepted recovery period for seasonal influenza of one week, with a complete recovery taking up to 10 days $[4,34]$.

Respondents who claimed they had seasonal influenza during the past year were asked to identify any symptoms related to their seasonal influenza. Most of the respondents identified more than one symptom. On average, respondents suffered 5.4 symptoms in Survey 1 and 7.2 symptoms in Survey 2. Table 5 provides the percentages based on the total number of symptoms mentioned for both surveys. The most common symptoms according to participants were cough, sore throat and fever. The least frequently mentioned symptoms were watery eyes, vomiting and nausea. 
Table 5

- A comparison (Survey 1 vs. Survey 2 ) between the symptoms related to the seasonal influenza, as mentioned by the survey respondents.

\begin{tabular}{|llll|}
\hline What were the symptoms? & Survey 1 & Survey 2 & Difference \\
\hline Cough & $15.5 \%$ & $13.1 \%$ & $-2.4 \%$ \\
\hline Sore throat & $14.1 \%$ & $13.1 \%$ & $-1.1 \%$ \\
\hline Fever & $12.2 \%$ & $7.8 \%$ & $-4.4 \%$ \\
\hline Runny or blocked nose & $10.6 \%$ & $12.8 \%$ & $2.1 \%$ \\
\hline Headache & $10.6 \%$ & $9.4 \%$ & $-1.3 \%$ \\
\hline Sneezing & $8.8 \%$ & $13.2 \%$ & $4.4 \%$ \\
\hline Muscle/joint pain & $5.3 \%$ & $11.7 \%$ & $6.3 \%$ \\
\hline Feeling tired or exhausted & $4.7 \%$ & $4.3 \%$ & $-0.5 \%$ \\
\hline Stomach ache & $3.3 \%$ & $0.5 \%$ & $-2.9 \%$ \\
\hline Diarrhoea & $3.0 \%$ & $2.7 \%$ & $-0.4 \%$ \\
\hline Shortness of breath & $2.7 \%$ & $1.9 \%$ & $-0.8 \%$ \\
\hline Loss of appetite & $2.7 \%$ & $1.8 \%$ & $-1.0 \%$ \\
\hline Chest pain & $2.6 \%$ & $2.4 \%$ & $-0.2 \%$ \\
\hline Nausea & $1.5 \%$ & $1.1 \%$ & $-0.4 \%$ \\
\hline Vomiting & $1.5 \%$ & $0.2 \%$ & $-1.3 \%$ \\
\hline Watery eyes & $0.6 \%$ & $4.2 \%$ & $3.6 \%$ \\
\hline Total & $100.0 \%$ & $100.0 \%$ & $0.0 \%$ \\
\hline Please note respondents were allowed to mention more than one symptom. & & & \\
\hline
\end{tabular}

Responses between both surveys were similar and within the $\pm 4.86 \%$ confidence interval, with the exception of the 'Muscle/joint pain' symptom where more participants reported it in Survey 2 than 1 (+6.3\% difference).

Respondents were also specifically asked if they had suffered from high body temperature. Out of the individuals who reported having seasonal influenza (including those who opted for the 'don't know' option), $64.2 \%$ claimed that they had high temperature in Survey 1 , while $55.0 \%$ in Survey 2, 22.4\% (42.4\% - Survey 2 ) did not and $13.4 \%$ (2.7\% - Survey 2 ) did not know. Furthermore, $68.7 \%$ visited a doctor due to their seasonal influenza (72.8\% - Survey 2$), 18.7 \%$ did not (27.2\% - Survey 2 ) and $12.7 \%$ did not remember (0\% - Survey 2 ). We also found that four out of every five respondents claiming to have contracted seasonal influenza took medicine to cure their symptoms (97.4\% - Survey 2 ), while $13.4 \%$ did not remember ( $0 \%$ - Survey 2 ). On the other hand, $20 \%$ of respondents in Survey 1 were hospitalised due to the seasonal influenza with only $4.0 \%$ in Survey 2. The hospitalised individuals spent an average of 6 nights (Survey 1 ) and 7.7 nights (Survey 2 ) at hospital.

The majority of respondents (54.5\% - Survey $1,24.9 \%$ - Survey 2 ) claimed that at least one additional member from their household had acquired seasonal influenza, excluding the respondent with 1.8 members on average reported in Survey 1 and 1.7 in Survey 2 . However, when also taking into account those who claimed (telephone respondents) that they had seasonal influenza, $61.1 \%$ of all Maltese households had at least one person with seasonal influenza (43.8\% - Survey 2$)$.

\section{Discussion}

In order to successfully predict and control infectious disease outbreaks and to design an appropriate public health response to an unfolding epidemic, we need to be able to accurately estimate the number of cases. There are different ways to achieve this, most notably medical records provided by GPs. The official Health Authority data are often assumed to give us the best estimation of the trends and they - unlike the telephone survey - can provide the real-time estimation of an unfolding epidemic [1]. However, none of these methods is capable of capturing all infection cases, a phenomenon known as underreporting. In this section, we compare the ability of different proxy measures to estimate the actual number of cases and argue that the telephone surveys can provide a valuable insight into the dynamics of seasonal influenza in Malta and particularly allow us to estimate the underreporting rate of official records. 


\section{Comparison between survey data and GP medical records}

We use the following proxy measures for the number of seasonal influenza cases:

1. Diagnosed ILI cases reported by the GPS

2. Respondents that reported seasonal influenza (survey data)

3. Respondents that reported ILI symptoms (survey data)

4. Respondents that reported high temperature (survey data)

5. Seasonal influenza cases in households (survey data)

\section{Number of cases}

The surveys were carried out amongst the Maltese population aged $18+$ years due to ethics requirements. In the lack of data for Malta showing a differential susceptibility for seasonal influenza among different age classes, the results were also assumed to be representative for the whole population of Malta. Thus, based on the results of Survey 1, the baseline total number of seasonal influenza cases in Malta between October 2014 and Mid-May 2015 can be estimated as ca. 130,000 cases. This number is obtained by multiplying the proportion of respondents in Survey 1 who reported having seasonal influenza (29.8\%) by the total population of 425,384 [35]. According to the data obtained from the Health Authority, an estimate of 32,000 seasonal influenza cases were reported by GPs in the same period. Based on this calculation, this implies that the official statistics provided by GPs is close to $25 \%$ reporting rate. After applying a similar calculation using Survey 2 data, this would result in a reporting rate equal to 18.1\% (based on $37.2 \%$ of respondents who reported having seasonal influenza), thus stressing consistency between both surveys (Table 6). However, this number likely underestimates the incidence, as people might have experienced seasonal influenza more than once. Using the 1.5 multiplier that we obtained from Survey 1 as the number of times the respondents felt they had seasonal influenza within a year, we obtain 195,000 cases (Table 6) and a reporting rate for the official records of $16.4 \%$.

\section{Number of respondents reporting at least one symptom}

Based on the results of Survey 1, the baseline total number of seasonal influenza cases in Malta between October 2014 and Mid-May 2015 can be estimated as ca. 360,000 cases. This number is obtained by multiplying the proportion of respondents in Survey 1 who reported having at least one symptom for seasonal influenza (84.6\%) by the total population of 425,384 [35]. Based on this calculation, this implies that the official statistics provided by GPs are as low as $9 \%$. After applying a similar calculation using Survey 2 data, this would result in a reporting rate equal to $8.5 \%$ (based on $80 \%$ of respondents who reported having at least one seasonal influenza symptom), again showing consistency between both surveys (Table 6). However, this is a clear overestimation of the total number of cases as we are assuming that all ILI symptomatic individuals eventually acquired the seasonal influenza, which is highly unlikely.

\section{Individuals' temperature}

One of the most significant symptoms of seasonal influenza is fever [33]. From all respondents, $28.6 \%$ claimed to have experienced fever during the year. If we consider this percentage as an estimate of the number of seasonal influenza cases, and again consider that individuals might have contracted seasonal influenza on average 1.5 times per year (as reported in Survey 1), we obtain a total of 180,000 seasonal influenza cases (Table 6) during 2014/2015. This result provides a reporting rate of $17.8 \%$. The same calculations carried out for the 2015/2016 survey yield a reporting rate of $20.7 \%$.

\section{Seasonal influenza cases in households (Survey data)}

According to Survey 1 results, $61.1 \%$ of all households in Malta had at least one household member with seasonal influenza, with an average of 1.8 people per household experiencing the seasonal influenza. According to the Maltese National Statistics Office (NSO), the total number of households in Malta is around 140,000 [35]. By using the latter data and taking into account that an individual might have experienced the seasonal influenza 1.5 times (Survey 1 ) during the same season, we can estimate that there were around 230,000 seasonal influenza cases (Table 6) during the $2014 / 2015$ season. Therefore, based on the GPs data, this result indicates that the reporting rate for the GP official records is $13.9 \%$. When applying the same methodology to the $2015 / 2016$ dataset, the reporting rate is $21.5 \%$. 
Table 6

- Number of cases for the two seasonal influenza seasons (2014/2015 and 2015/2016)

based on 4 different variables.

\begin{tabular}{|lll|}
\hline & $\begin{array}{l}\text { 2014/2015 } \\
\text { (Survey 1) } \\
\text { Number of influenza cases }\end{array}$ & $\begin{array}{l}\text { 2015/2016 } \\
\text { (Survey 2) }\end{array}$ \\
\hline Seasonal influenza cases & 195,000 & 200,000 \\
\hline Symptomatic cases & 360,000 & 340,000 \\
\hline Individual's temperature & 180,000 & 140,000 \\
\hline Influenza cases in households & 230,000 & 135,000 \\
\hline
\end{tabular}

We have thus shown that based on the $2014 / 2015$ survey, the reporting rate of the official GP records varied between $13.9 \%$ and $17.8 \%$. For the $2015 / 2016$ season, the reporting rate varied between $14.5 \%$ and $21.5 \%$. The lowest estimated reporting rates of $8.9 \%$ (Survey 1 ) and $8.5 \%$ (Survey 2) were based on the assumption that any of the above-mentioned symptoms corresponded to the individual having seasonal influenza.

Using the survey data, we were able to estimate the number of individuals in Malta who had acquired seasonal influenza during $2014 / 2015$ season as between 120,000 and 150,000 . Thus, between $28 \%$ and $36 \%$ of the Maltese citizens had seasonal influenza during the $2014 / 2015$ period, while for the $2015 / 2016$ season, this varied between 100,000 (24.5\%) and 160,000 (37.2\%) individuals. According to the CDC, seasonal influenza in the United States affects between $5 \%$ and $20 \%$ of the total population [36]. In Finland, it was estimated that $6 \%$ were infected during the first wave of the $2009 / 2010$ pandemic season and $3 \%$ during the second wave [37]. None of these estimates were based on crosssectional surveys, but rather from on-line data [36] and national surveillance data [37]. However, one cannot really directly compare Malta's incidence rate with other countries, as Malta is an island state and one of the most densely populated countries in the world [38]. Given that physical contact between people is more likely to occur in Malta, then the transmission rate of seasonal influenza will be greater than those of the US and Finland [39].

\section{Comparison of different sources of data}

Data gathered from these surveys shed more light on different characteristics of seasonal influenza such as symptoms, months in which respondents claimed to have several ILI symptoms, and months when they thought they acquired the seasonal influenza. The latter two variables can be directly compared with the data obtained by a more traditional route of GP reporting (Fig. 1). In order to translate the surveys' results into a proxy measure of the number of cases, we will use:

1. The monthly occurrences of ILI symptoms as stated by the survey respondents. The survey question related to this analysis was, 'When did your symptoms appear for the above during the past year?' 'Above' in this question corresponds to the list of symptoms as defined in Table 2 .

2. The monthly occurrences of seasonal influenza as stated by the survey respondents. The survey question related to this analysis was, 'If 'Yes', when did you have the seasonal influenza?' The 'Yes' reply corresponds to the respondents who claimed that they had experienced seasonal influenza during a one-year period.

There is a good agreement between the shape of the time dependence of monthly reports of the ILI symptomatic cases (as stated by the survey respondents) (Fig. 1a) and the 2014/2015 diagnosed ILI cases (GPs reported data) (Fig. 1e). This agreement is reflected by a strong linear correlation $(r=0.90 ; p$-value $=0.002)($ Table 7$)$. Although the survey data were collected retrospectively, the respondents appear to remember the actual months when they had the symptoms. Interestingly, the last three months of the survey data registered a higher number of symptoms reported in Survey 1, when compared with the observed diagnosed ILI data (Fig. 1a).

Similarly, in Fig. 1c, the monthly reports of seasonal influenza cases, as stated by the survey respondents, show good agreement with the seasonal influenza cases as reported by the GPs (Fig. 1e), with high correlation $(r=0.88$, $p$-value $=0.004)($ Table 7$)$. The data for the last three months show lower values than the one based on the symptoms (compare Fig. 1a and 1c).

The two survey variables, symptoms and self-diagnosis, are also correlated $(r=0.85, p=0.008)$. However, only around $30 \%$ of participants claimed they had seasonal influenza, while around $84.6 \%$ claimed that they had any of the above ILI symptoms. Hence, based on these results, it is likely that respondents have a different perception of the definition of seasonal influenza. Therefore, illness perceptions and health beliefs can be rather subjective, although are important predictors for health utilization $[9,10]$. 
Table 7

- Correlation analysis for the three variables related to the months of the influenza symptoms (2014/2015 season). 'GPs_Influenza' is the diagnosed seasonal influenza individuals collected by the GPs, while 'Survey_Symptoms' variable is the monthly occurrences of the Influenza-like IIIness (ILI) symptomatic cases as stated by the survey respondents and 'Survey_Influenza' variable is the monthly occurrences of the seasonal influenza cases as stated by the survey respondents.

\begin{tabular}{|llll|}
\hline & GPs_Influenza & Survey_Symptoms & Survey_Influenza \\
\hline GPs_Influenza & 1 & 0.899 & 0.882 \\
& & $(p-v a l u e=0.002)$ & $(p-v a l u e=0.004)$ \\
\hline Survey_Symptoms & 1 & 0.846 \\
& & $(p-v a l u e=0.008)$ \\
\hline Survey_Influenza & & 1 \\
\hline
\end{tabular}

Similar results were obtained for the $2015 / 2016$ survey (Fig. 1b, $d$ and $f$ and Table 8 ). The months in which the respondents reported the symptoms or self-diagnosed seasonal influenza are similar to the months that were associated with high levels of ILI cases as recorded by the GPs (compare Fig. 1b and d with f).

Table 8

- Correlation analysis for the three variables related to the months of the influenza symptoms (2015/2016 season).

\begin{tabular}{|c|c|c|c|}
\hline & GPs_Influenza & Survey_Symptoms & Survey_Influenza \\
\hline \multirow[t]{2}{*}{ GPs_Influenza } & 1 & 0.935 & 0.929 \\
\hline & & $(p$-value $=0.002)$ & $(p$-value $=0.002)$ \\
\hline \multirow[t]{2}{*}{ Survey_Symptoms } & & 1 & 0.890 \\
\hline & & & $(p-$ value $=0.007)$ \\
\hline \multicolumn{3}{|l|}{ Survey_Influenza } & 1 \\
\hline
\end{tabular}

Google Trends

In addition, we compared both the results of the surveys and the GP notifications with the Google Trends records using the key words 'Influenza', 'Flu' and 'Cold' in both Maltese and English language for searches that were carried out in Malta. The Google searches (Figs. 1g, $1 \mathrm{~h}$ ) all show a peak in January, as seen in other data, but the dynamics in other months does not capture the rise in cases in OctoberDecember and fall in February-May periods (Figs. 1e, 1f).

\section{Symptoms and medical condition}

Both surveys show consistent data related to the general medical conditions of the individuals, with the most common symptoms similar across both surveys. The main differences lie in the levels of self-reporting of seasonal influenza (30\% in Survey 1 and $37 \%$ in Survey 2 ) and in the number of times the respondents felt they had experienced seasonal influenza (1.5 times in Survey 1 and 1.28 in Survey 2 ). Furthermore, according to the Survey 2, a lower number of Maltese households registered seasonal influenza cases than in Survey 1 . We already showed through the survey results (and GPs data) that the number of infected individuals varies between the two seasons, and this could be attributed to the climate conditions [40].

\section{Summary of advantages and disadvantages for different methods}

Cross-sectional surveys carried out via telephone are easy to conduct and can include extensive information about seasonal influenza (Table 9). However, most data are based on self-diagnosis and self-reporting. Although GPs data are based on medical diagnosis and experts' opinion as well as being available in real time, the reporting efficiency can be quite low. Google Trends provide an indication about the peak of the outbreak but it fails to provide information about the severity of the same outbreak. Online surveys and serological data in relation to the seasonal influenza are not available in Malta, but the online surveys introduce additional bias as they capture only a subsection of the population. 
Table 9

- Different sources of information in relation to the seasonal influenza.

\begin{tabular}{|c|c|c|c|}
\hline $\begin{array}{l}\text { Source of } \\
\text { Data }\end{array}$ & Type of Data & Advantages & Disadvantages \\
\hline $\begin{array}{l}\text { Telephone } \\
\text { Surveys }\end{array}$ & $\begin{array}{l}\text { - Influenza Symptoms } \\
\text { - Number of influenza cases } \\
\text { - Number of hospitalisation } \\
\text { due to influenza } \\
\text { - Respondents' medical } \\
\text { information } \\
\text { - Other dynamics in relation } \\
\text { to the influenza }\end{array}$ & $\begin{array}{l}\text { - Easy to collect } \\
\text { - Has the possibility to include extensive } \\
\text { information about the outbreak } \\
\text { - Data can be compared against non-influenza } \\
\text { individuals }\end{array}$ & $\begin{array}{l}\text { - Relies on self-diagnosis and self- } \\
\text { reporting }\end{array}$ \\
\hline $\begin{array}{l}\text { GP } \\
\text { reporting }\end{array}$ & $\begin{array}{l}\text { - Consultations } \\
\text { - Diagnosed }\end{array}$ & $\begin{array}{l}\text { - Medical data } \\
\text { - Weekly data }\end{array}$ & $\begin{array}{l}\text { - Consultation include other cases } \\
\text { - Average of } 6 \text { doctors out of } 300 \\
\text { reporting cases; low coverage }\end{array}$ \\
\hline $\begin{array}{l}\text { Google } \\
\text { Trends }\end{array}$ & - Search by popularity & - Quick and easy to collect & $\begin{array}{l}\text { - Data relies on internet connected } \\
\text { individuals only } \\
\text { - Data does not tell you the severity of the } \\
\text { outbreak }\end{array}$ \\
\hline $\begin{array}{l}\text { Online } \\
\text { surveys }\end{array}$ & Not available in Malta & & \\
\hline $\begin{array}{l}\text { Serological } \\
\text { data }\end{array}$ & Not available in Malta & & \\
\hline
\end{tabular}

\section{Conclusions}

There are limited studies that focus on analysing in depth factors related to the quality of seasonal influenza reporting and details of the symptoms. Throughout this research study, we demonstrated that telephone surveys are a reliable way to collect epidemiological data and can be used to estimate the total number of seasonal influenza cases. They also give a unique insight into other important factors like selfperception and distribution of symptoms among the population. We have also showed that when two surveys are carried out at different times in relation to the seasonal influenza outbreak (either after the outbreak, or during the tail of the epidemic), the results from the two surveys are comparable. Thus, we claim that this novel method helps to provide another useful data set for medical statistics, in addition to those provided by the official notifications by GPs and by Google Trends. The collected information can be used to improve the epidemiological modelling related to the seasonal influenza and other infectious diseases, and thus can contribute to public health initiatives [41]. In particular, we used the survey data to estimate the reporting rate of the GP system that forms the basis for official records and hence underlies the key decisions taken by the Health Authorities. We showed that based on the two seasons, the reporting rate in Malta varies between $14 \%$ and $22 \%$.

However, we need to treat such results with caution. To a certain extent, we are comparing self-diagnosis of individuals against the GPs seasonal influenza diagnosis. Hence, the baseline for both numbers is most likely not the same. The self-diagnosis provides an estimate of the actual seasonal influenza cases based on personal perception. Nevertheless, there are several indicators throughout the survey that have shown that these results are a true representation of the actual population. The monthly data between the survey and GPs data (Fig. 1) match fairly well, thus providing an extra level of confidence that the respondents were accurately remembering their medical history for the past year. Participation and response rates in epidemiological surveys are very important [42], therefore the right methodologies are needed to ensure that the response rate is satisfactory [42]. Such studies can already contain certain elements of bias, since several responses are based on the respondents' medical knowledge. For a noticeable number of questions, respondents often base their judgement on self-medical diagnosis. This is considered an important element in epidemiological studies as it supports pandemic control strategies through self-management practices and the reduction of visits to healthcare facilities, thereby aiding to contain viral spread [43]. Self-reports have been compared to patients' electronic medical records [44] in order to examine the accuracy of self-reporting vaccination status. Nonetheless, there is limited evidence about the accuracy of self-reports of seasonal influenza, particularly during pandemics [43], warranting further in-depth analysis, as is found in this study.

Most of the results related to the symptoms are in accordance to the findings of the UK flu survey [24], which reports the most common symptoms as runny nose, cough, sneezing, headache, sore throat and feeling tired. However, the UK survey data are biased towards those individuals that have frequent internet access and so can skew the results towards those with a higher level of education [24]. 
Further work is warranted to understand to what extent these surveys can contribute to our understanding of disease diagnosis if they were to be conducted during an actual outbreak. Running a series of cross-sectional surveys during various stages of the seasonal influenza outbreak might provide further understanding of people's perceptions of seasonal influenza, and probe deeper into whether the survey results are timedependent. Furthermore, scientific surveys can provide detailed information to understand the real notion of seasonal influenza, and to offer an opportunity to improve the prior information for future epidemiological modelling, and provide even further refinements beyond this analysis. Furthermore, survey findings can be tested using other observed datasets to examine their validity in the context of epidemiological studies. All this information can aid in designing a package of different sources of information in support to the prediction of current and future influenza outbreaks.

In summary, we show that a cross-sectional telephone survey is a valid way of gathering post-hoc epidemiological data as it produces results that are comparable with the practitioner reports. Thus, our study provides further proof of the validity of the usual assumptions made in infectious disease modelling of a proportionality of observed to actual cases. However, we also show that through telephone surveys we can gain deeper insight into population self-perception of seasonal influenza and its symptoms.

\section{Declarations}

\section{Ethics approval and consent to participate:}

Ethics approval was obtained via an application to the Psychology Ethics Committee at University of Stirling (28th August 2015). All participants gave verbal informed consent before participating in the study.

\section{Consent for publication:}

Not applicable

Availability of data and materials:

Data supporting the conclusions of this study are included within the manuscript. The raw datasets analysed during the current study are available from the corresponding author on reasonable request.

\section{Competing interests:}

The authors declare that they have no competing interests regarding the publication of this paper.

\section{Funding:}

Self-funding.

\section{Authors' contributions:}

VM conceived the study and wrote the manuscript. VM supervised all aspects of its conduction. VM conducted data analysis and interpretation of data. DM, PM and AK critically reviewed and revised the manuscript. All authors helped to review drafts of the manuscript. VM read and approved the final manuscript.

\section{Acknowledgements:}

This study was developed as part of the activities of the PhD offered by the University of Stirling in Scotland.

\section{References}

[1] V. Marmara, A. Cook, A. Kleczkowski, Estimation of force of infection based on different epidemiological proxies: $2009 / 2010$ Influenza epidemic in Malta, Epidemics, 9 (2014) 52-61.

[2] Google.com, Google trends, United States, 2017, URL:https://trends.google.com/trends/

[3] NHS, National Health Service (UK), Symptoms of flu, 2015, URL: http://www.nhs.uk/Conditions/Flu/Pages/Symptoms.aspx. 
[4] WHO, World Health Organization, Influenza (Seasonal), World Wide Web electronic, 2014, URL:

http://www.who.int/mediacentre/factsheets/fs211/en/.

[5] CDC, Centers for Disease Control and Prevention (USA), Key Facts about Influenza (Flu) \& Flu Vaccine, 2015, URL:

http://www.cdc.gov/flu/keyfacts.htm\#whatis.

[6] CDPH, California Department for Public Health (USA), H1N1 Influenza, 2009, URL:

https://www.cdph.ca.gov/HealthInfo/h1n1flufaqs/Pages/H1N1fluFAQs-01-GenInfo.aspx\#01.06.

[7] E.Y. Chan, C.K. Cheng, G.C. Tam, Z. Huang, P.Y. Lee, Willingness of future A/H7N9 influenza vaccine uptake: A cross-sectional study of Hong Kong community, Vaccine, 33 (2015) 4737-4740.

[8] P. Mangtani, E. Breeze, S. Stirling, S. Hanciles, S. Kovats, A. Fletcher, Cross-sectional survey of older peoples' views related to influenza vaccine uptake, BMC Public Health, 6:249 (2006) DOI: 10.1186/1471-2458-6-2492011.

[9] A. Pfeil, M. Mutsch, C. Hatz, T.D. Szucs, A cross-sectional survey to evaluate knowledge, attitudes and practices (KAP) regarding seasonal influenza vaccination among European travellers to resource-limited destinations, BMC Public Health, 10:402 (2010) DOI:10.1186/1471-245810-402

[10] G.E. Khoury, P. Salameh, Influenza Vaccination: A Cross-Sectional Survey of Knowledge, Attitude and Practices among the Lebanese Adult Population, International Journal of Environment Research and Public Health, 12 (2015) 15486-15497.

[11] J. Zhang, A.E. While, I.J. Norman, Nurses' knowledge and risk perception towards seasonal influenza and vaccination and their vaccination behaviours: A cross-sectional survey, International Journal of Nursing Studies, 48 (2011) 1281-1289.

[12] J. Mereckiene, S. Cotter, A. Nicoll, P. Lopalco, T. Noori, J.T. Weber, F. D’Ancona, D. Levy-Bruhl, L. Dematte, C. Giambi, P, Valentiner-Branth, I. Stankiewicz, E. Appelgren, D. O'Flanagan, the VENICE project gatekeepers group, Seasonal influenza immunisation in Europe. Overview of recommendations and vaccination coverage for three seasons: pre-pandemic (2008/09), pandemic (2009/10) and post-pandemic (2010/11), Euro Surveillance, 19(16) (2014) URL: http://www.eurosurveillance.org/ViewArticle.aspx?Articleld=20780.

[13] J. Yang, M. Jit, K.S. Leung, Y. Zheng, L. Feng, L. Wang1, E.H.Y. Lau, J.T. WU, H. Yu, The economic burden of influenza-associated outpatient visits and hospitalizations in China: a retrospective survey, Infectious Diseases of Poverty, 4:44 (2015) D0I: 10.1186/s40249-015-0077-6.

[14] B.Y. Lee, K. Bacon, J.M. Donohue, A.E. Wiringa, R.R. Bailey, R.K. Zimmerman, From the Patient Perspective: the Economic Value of Seasonal and H1N1 Influenza Vaccination, Vaccine, 29(11) (2011) 2149-2158.

[15] C. Wu, J. Lu, M.H. Wang, X. Lv, Y. Chen, H. Kung, B. Zee, X. Cheng, M. He, Cross Sectional Survey of Influenza Antibodies before and during the 2009 Pandemic in Shenzhen, China, PLoS ONE, 8(1) (2013) DOI: 10.1371/journal.pone.0053847.

[16] X. Tan, L.Yuan, J. Zhou, Y. Zheng, F. Yang, Modeling the initial transmission dynamics of influenza A H1N1 in Guangdong Province, China, International Journal of Infectious Diseases, 17 (2013) e479-e484.

[17] V.J. Lee, M.I. Chen, J. Yap, J. Ong, W. Lim, R.T.P. Lin, I. Barr, J.B.S. Ong, T.M. Mak, L.G. Goh, Y.S. Leo, P.M. Kelly, A.R. Cook, Comparability of Different Methods for Estimating Influenza Infection Rates Over a Single Epidemic Wave, American Journal of Epidemiology, 174(4) (2011) 468-478.

[18] P.A. Oria, G. Arunga, E. Lebo, J.M. Wong, G. Emukule, P. Muthoka, N. Otieno, D. Mutonga, R.F. Breiman, M.A Katz, Assessing parents' knowledge and attitudes towards seasonal influenza vaccination of children before and after a seasonal influenza vaccination effectiveness study in low-income urban and rural Kenya, 2010-2011, BMC Public Health, 13:391 (2013) URL: http://www.biomedcentral.com/1471$2458 / 13 / 391$.

[19] F. Anday, P. Crepey, A. Kieffer, N. Salez, A.A. Abdo, F. Carrat, A. Flahault, X. Lamballerie, Determinants of individuals' risks to 2009 pandemic influenza virus infection at household level amongst Djibouti city residents - A CoPanFlu cross-sectional study, Virology Journal, 11:13 (2014) URL: http://www.virology.j.com/content/11/1/13.

[20] S.E. Soh, A.R Cook, M.I.C. Chen, V.J. Lee, J.L. Cutter, V.T.K. Chow, N.W.S. Tee, R.T.P. Lin, W. Lim, I.G. Barr, C. Lin, M.C. Phoon, L.W. Ang, S.K. Sethi, C.Y. Chong, L.G. Goh, D.L.M. Goh, P.A. Tambyah, K.C. Thoon, Y.S. Leo, S.M. Saw, Teacher led school-based surveillance can allow accurate tracking of emerging infectious diseases - evidence from serial cross-sectional surveys of febrile respiratory illness during the $\mathrm{H} 1 \mathrm{~N} 1$ 2009 influenza pandemic in Singapore, BMC Infectious Diseases, 12:336 (2012) URL: http://www.biomedcentral.com/1471-2334/12/336. 
[21] WHO, Pandemic (H1N1) 2009 - update 100, 2010 URL https://www.who.int/csr/don/2010_05_14/en/ [22] A. Ishak , D. Tee, I. Nawmar, L.K. Pang, N. Ruslan, N. Che Mansor, L. Gam, H1N1 Influenza: A Viral Infection, Webmed Central Infectious Diseases, 2(12) (2011) WMC002736.

[23] C. Reed, F.J. Angulo, D.L. Swerdlow, M. Lipsitch, M.I. Meltzer, D. Jernigan, and L. Finelli, Estimates of the Prevalence of Pandemic (H1N1) 2009, United States, April-July 2009, Nature Communication, 4 (2013) 2004-2007.

[24] UK flusurvey, London School of Hygiene and Tropical Medicine and Public Health England, 2016 URL: https://flusurvey.org.uk/en/.

[25] A. Camacho, K. Eames, A. Adler, S. Funk, J. Edmunds, Estimate of the quality of life effect of seasonal influenza infection in the UK with the internet-based Flusurvey cohort: an observational cohort study, Lancet, 383(3) (2013) 8-8.

[26] A.J. Adler, K.T.D. Eames, S. Funk, W.J. Edmunds, Incidence and risk factors for influenza-like-illness in the UK: online surveillance using Flusurvey, BMC Infectious Diseases, 14:232 (2014) URL: http://www.biomedcentral.com/1471-2334/14/232.

[27] Gripenet, Department of Epidemiology, National Institute of Health Dr. Ricardo Jorge, Lisbon Portugal, 2016 URL: http://www.gripenet.pt/pt/.

[28] GrippeNet, Institute Pierre Louis, France, 2016 URL: https://www.grippenet.fr/fr/.

[29] Gripenet, Complex Systems and Networks Lab, Institute for Biocomputation and Physics of Complex Systems (BIFI), Campus Rio Ebro, University of Zaragoza, Spain, 2016 URL: https://www.gripenet.es/es/.

[30] P.J. Birrell, G. Ketsetzis, N.J. Gay, B.S. Cooper, A.M. Presanis, R.J. Harris, A. Charlett, X. Zhang, P.J. White, R.G. Pebody, D.D Angelis, Bayesian modeling to unmask and predict influenza A/H1N1pdm dynamics in London, PNAS, 108(45) (2008) 18238-18243.

[31] J.L. Malone, M. Madjid, S.W. Casscells, Telephone Survey to Assess Influenza-like Illness, United States, 2006, Emerging Infectious Diseases, 14(1) (2008) URL: www.cdc.gov/eid.

[32] National Statistics Office, Malta (NSO), 'Demographic Review 2013', 2015, URL:

https://nso.gov.mt/en/publicatons/Publications_by_Unit/Documents/C3_Population_and_Tourism_Statistics/Demographic_Review_2013.pdf.

[33] WebMD, Cold, Flu, \& Cough Health Center, World Wide Web electronic, 2016 URL: http://www.webmd.com/cold-and-flu/fluguide/advanced-reading-types-of-flu-viruses\#4.

[34] PAGB, Proprietary Association of Great Britain, World Wide Web electronic, 2016 URL:

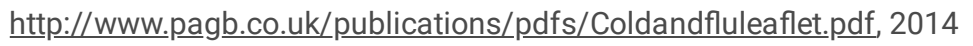

[35] S. Galea, M. Tracy, Participation Rates in Epidemiologic Studies, Ann Epidemiology, 17 (2007) 643-653.

[36] A. Jutel, M.G. Baker, J. Stanley, Q.S. Huang, D. Bandaranayake, Self-diagnosis of influenza during a pandemic: a cross-sectional survey, BMJ Open, 1 (2011) DOI: 10.1136/bmjopen-2011-000234

[37] S.J. Rolnick, E.D. Parker, J.D. Nordin, B.D. Hedbloma, F. Wei, T. Kerby, J.M. Jackson, A.L Crain, G. Euled, Self-report compared to electronic medical record across eight adult vaccines: Do results vary by demographic factors?, Vaccine, 31(37) (2013) 3928-3935.

[38] National Statistics Office, Malta (NSO), 'Malta in Figures 2014', 2015 URL: http://nso.gov.mt/en/publicatons/Pages/Publications-byDate.aspx

[39] K.S. Hickmann, G. Fairchild, R. Priedhorsky, N. Generous, J. M. Hyman, A. Deshpande, S.Y. Del Valle, Forecasting the 2013-2014 Influenza Season using Wikipedia, Plos Computational Biology, 11(5) (2015) DOI: 10.1371/journal.pcbi.1004239.

[40] M. Shubin, A. Lebedev, O. Lyytikäinen, K. Auranen, Revealing the True Incidence of Pandemic A (H1N1)pdm09 Influenza in Finland during the First Two Seasons-An Analysis Based on a Dynamic Transmission Model, Plos Computational Biology, 11(3) (2016) DOI: 10.1371/journal.pcbi.1004803.

[41] World Population Review, 2019 URL: http://worldpopulationreview.com/countries/malta-population/

[42] J. Mossong, N. Hens, M. Jit , P. Beutels, K. Auranen, R. Mikolajczyk, M. Massari, S. Salmaso, G.S. Tomba, J. Wallinga, J. Heijne, M. Sadkowska-Todys, M. Rosinska, W.J. Edmunds, Social Contacts and Mixing Patterns Relevant to the Spread of Infectious Diseases, PLOS Medicine, 5(3) (2008) 381-391. 
[43] C. K. Irwin, K. J. Yoon, C. Wang, S. J. Hoff, J. J. Zimmerman, T. Denagamage, and A. M. O'Connor, Using the Systematic Review Methodology To Evaluate Factors That Influence the Persistence of Influenza Virus in Environmental Matrices, Applied and Environmental Microbiology, 77(3) (2011) 1049-1060.

[44] Kleczkowski A, Hoyle A, McMenemy P. One model to rule them all? Modelling approaches across OneHealth for human, animal and plant epidemics. Philosophical Transactions of the Royal Society B 374: 20180255. (2019) http://dx.doi.org/10.1098/rstb.2018.0255

\section{Figures}
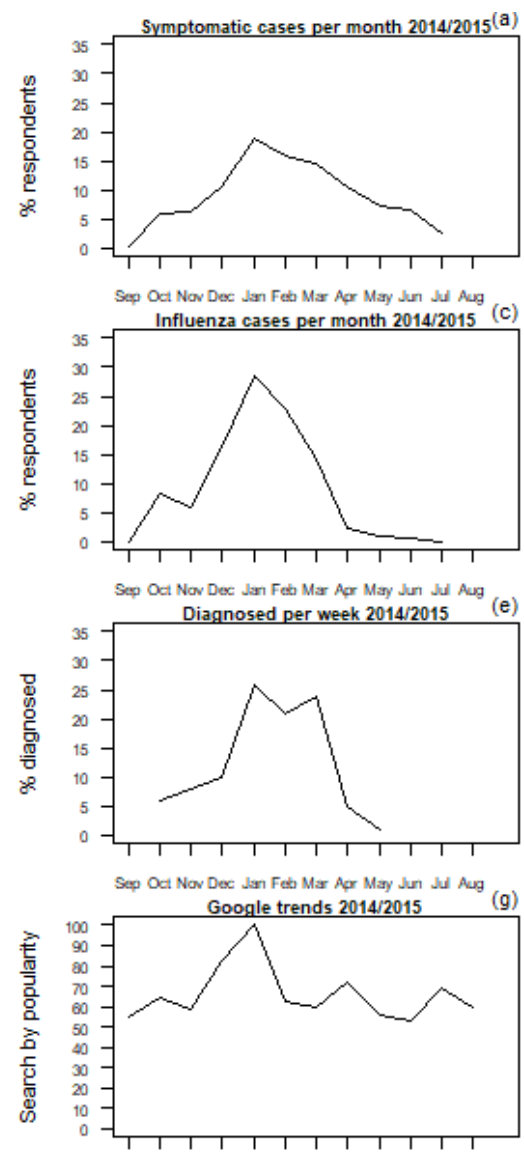

Sepp Oet Now Dec Jan Feb Mar Acar May Jun Jui Aug
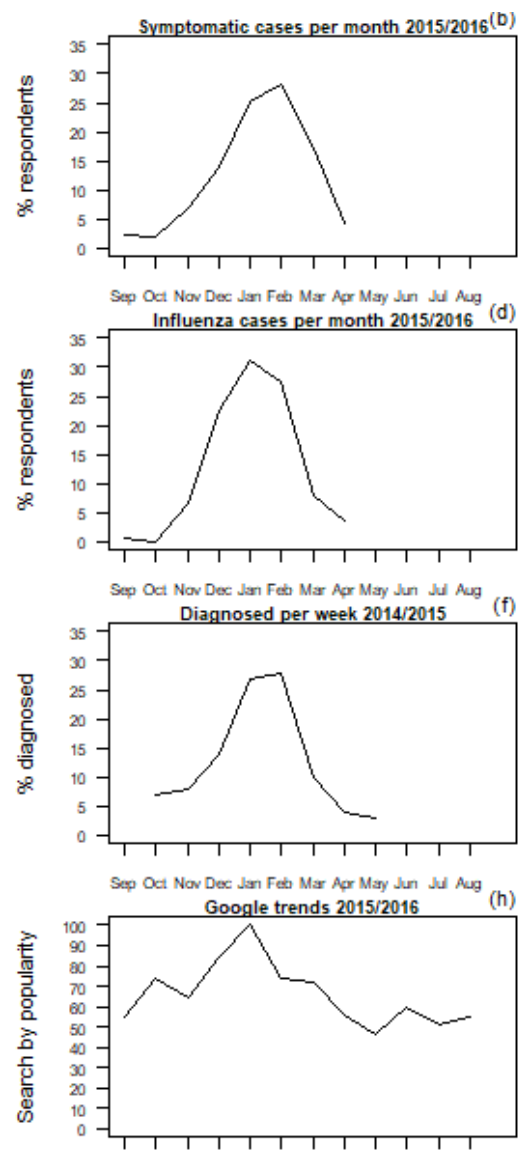

Sepp Oat Now Dec Jan Feb Max Aq̣ May Jun Jui Aug

\section{Figure 1}

(a) \% of the monthly occurrences of the 2014/2015 Influenza-like Illness (ILI) symptomatic cases as stated by the survey respondents from the total monthly occurrences mentioned, (c) \% of the monthly occurrences of the 2014/2015 seasonal influenza cases as stated by the survey respondents from the total monthly occurrences mentioned, (e) \% of the monthly GP diagnosed ILI cases for the 2014/2015 season from the total number of diagnosed ILIs throughout the whole season, (g) search by popularity of the Google search trends for the 2014/2015 season using the key words 'Influenza', 'Flu' and 'Cold' in both Maltese and English language for searches that were carried out in Malta. Charts (b), (d), (f) and (h) have the same definitions as (a), (c), (e) and (g) respectively but for the 2015/2016 season.

\section{Supplementary Files}

This is a list of supplementary files associated with this preprint. Click to download.

- Appendix1SurveyResults.docx 